\title{
Atherosclerosis and Lipids
}

\author{
Shinjiro SuzukI
}

The National Institute of Nutrition

Though lipids have generally been restricted in the case of atherosclerosis, vegetable oils have no rising effect on serum cholesterol level, moreover, rice bran oil, safflower oil, wheat germ oil, corn oil and sunflower oil lower distinctly its level.

This cholesterol lowering effect is attributed to the unsapponifiable matters (rice bran oil) and form of glycerides as well as linoleic acid content. 